CERN-TH.7331/94

\title{
A DIRECT TEST OF PERTURBATIVE QCD AT SMALL $x$
}

\author{
Richard D. Ball* and Stefano Forte $\dagger$ \\ Theory Division, CERN, \\ CH-1211 Genève 23, Switzerland.
}

\begin{abstract}
We show that recent data from HERA on the proton structure function $F_{2}$ at small $x$ and large $Q^{2}$ provide a direct confirmation of the double asymptotic scaling prediction of perturbative QCD. A linear rise of $\ln F_{2}$ with the scaling variable $\sigma$ is observed throughout the kinematic region probed at HERA, and the measured slope is in excellent agreement with the QCD prediction. This provides a direct determination of the leading coefficient of the beta function. At large values of the scaling variable $\rho$ the data display a small but statistically significant scaling violation.
\end{abstract}

Submitted to: Physics Letters B

CERN-TH.7331/94

June 1994

* On leave from a Royal Society University Research Fellowship.

$\dagger$ On leave from INFN, Sezione di Torino, Italy. 
Perturbative QCD predicts that at sufficiently large $t \equiv \ln Q^{2} / \Lambda^{2}$ and small $x$ the nucleon structure function $F_{2}$ should exhibit double scaling in the two variables

$$
\sigma \equiv \sqrt{\ln \frac{x_{0}}{x} \ln \frac{t}{t_{0}}}, \quad \rho \equiv \sqrt{\ln \frac{x_{0}}{x} / \ln \frac{t}{t_{0}}}
$$

provided only that the nonperturbative input to the perturbative evolution is sufficiently soft. We have shown [1] that this prediction is indeed confirmed by the first measurements of $F_{2}^{p}$ performed at HERA[2,3]. In fact, it turns out that not only most of the HERA data, but even some of the older data from the NMC [4], lie well inside the asymptotic regime, suggesting that the starting scale $t_{0} \equiv \ln Q_{0}^{2} / \Lambda^{2}$ for the perturbative evolution should be little more than $Q_{0}^{2} \sim 1 \mathrm{GeV}^{2}$. A significantly enlarged set of measurements of $F_{2}^{p}$ has now become available [5,6], which makes it possible to test double scaling more quantitatively. Specifically, the slope of the linear rise of $\ln F_{2}$ in the scaling variable $\sigma$ can be reliably measured, and turns out to be in excellent agreement with the QCD prediction, thus giving a direct empirical determination of the leading coefficient $\beta_{0}$ of the QCD beta-function. We also find that there is now evidence for scaling violation at large $\rho$.

Double asymptotic scaling follows from a computation [7] of the asymptotic form of the structure function $F_{2}^{p}(x ; t)$ at small $x$ based on the use of the operator product expansion and renormalization group at leading perturbative order. It thus relies only on the assumption that any increase in $F_{2}^{p}(x ; t)$ at small $x$ is generated by perturbative QCD evolution, rather than being due to some other (nonperturbative) mechanism manifested by an increase in the starting distribution $F_{2}^{p}\left(x ; t_{0}\right)$. The resulting asymptotic behaviour takes the form

$$
F_{2}^{p}(\sigma, \rho) \sim N f\left(\frac{\gamma}{\rho}\right) \frac{\gamma}{\rho} \frac{1}{\sqrt{\gamma \sigma}} \exp \left[2 \gamma \sigma-\delta\left(\frac{\sigma}{\rho}\right)\right]\left[1+O\left(\frac{1}{\sigma}\right)\right]
$$

where $\gamma \equiv 2 \sqrt{N_{c} / \beta_{0}}, \beta_{0}=\frac{11}{3} N_{c}-\frac{2}{3} n_{f}, \delta \equiv\left(1+\frac{2 n_{f}}{11 N_{c}^{3}}\right) /\left(1-\frac{2 n_{f}}{11 N_{c}}\right)$, and the unknown function $f$, which depends on the details of the starting distribution, tends to one for sufficiently small values of its argument. $N$ is an a priori undetermined normalization factor.

In [1] we derived (2) by noting that at small- $x$ the one loop QCD evolution equations reduce to wave equations, which propagate the parton distribution functions from their boundary values at $t=t_{0}$ and $x=x_{0}$ to larger values of $t$ and smaller values of $x$. Since the propagation is unstable, away from the boundaries an exponential increase with $\sigma$ of the form (2) inevitably arises, provided only that the small- $x$ behaviour of the starting 
distributions at $t_{0}$ is sufficiently soft (which in practice means that if $f_{s}(x ; t)$ is a singlet parton distribution function, $x^{1+\lambda} f_{s}\left(x ; t_{0}\right) \rightarrow 0$ as $x \rightarrow 0$ for any $\left.\lambda \lesssim 0.2\right)$. The behaviour (2) is thus a rather clean prediction of perturbative QCD, in so far as it is independent of the details of the (soft) nonperturbative parton distributions which are input at $t_{0}$, provided that at small $x$ these conform to expectations based on Regge theory. The asymptotic behaviour can be shown [1] to set in rather rapidly as $\sigma$ increases in a region not too close to the boundaries, i.e. when $\rho$ is neither too large nor too small.

In order to compare the data for $F_{2}^{p}$ with the prediction (2) we rescale the measured values of $F_{2}$ by a factor

$$
R_{F}^{\prime}(\sigma, \rho)=R \exp \left(\delta(\sigma / \rho)+\frac{1}{2} \ln \sigma+\ln (\rho / \gamma)\right)
$$

to remove the part of the leading subasymptotic behaviour which can be calculated in a model independent way. 1 Then $\ln \left[R_{F}^{\prime} F_{2}\right]$ is predicted to rise linearly with $\sigma$, independently of $\rho$ (when $\rho$ is large), with slope

$$
2 \gamma=12 / \sqrt{33-6 n_{f} / N_{c}}=2.4
$$

if $n_{f}=4$ as in the HERA kinematic range. The model-dependent subasymptotic behaviour due to the function $f$ can be eliminated by cutting all points with subasymptotically small $\rho$; the scaling analysis of Ref.[1] (see fig. 2 below) suggests that we place the cut at $\rho^{2}=2$.

All the available experimental data [4,5],6] for $F_{2}^{p}$ which pass this cut are plotted in fig. 1. The predicted linear rise in $\sigma$ is spectacularly confirmed, providing clear evidence that in the region $\sigma^{2}>1, \rho^{2}>2$ the asymptotic behaviour (2) has set in. Indeed, the scaling actually sets in rather precociously: even the NMC data down to $\sigma \sim 0.7$ seem to be rising linearly, with possibly an indication of a systematic normalization mismatch of around $10 \%$ between the NMC and the HERA determinations of $F_{2}$.

Fitting a straight line to all 80 HERA points in the plot yields a $\chi^{2}$ of 66 , and a gradient $2 \gamma_{\text {exp }}=2.37 \pm 0.16$, in perfect agreement with the QCD prediction eq.(止). Turning this into a measurement of the leading coefficient of the beta-function gives (with $N_{c}=3$ ) $\beta_{0}=8.6 \pm 1.1$ (to be compared with $25 / 3$ for $n_{f}=4$ ). This is a direct, model independent, and highly nontrivial test of the perturbative dynamics of asymptotically free nonabelian gauge theory.

1 The constant rescaling factor $R$ may of course be chosen arbitrarily; here we choose $R=8.1$, so that the normalization of the figures is the same as in [1]. 


\begin{tabular}{|c|l|l|r|}
\hline & \multicolumn{1}{|c|}{$N_{s}$} & \multicolumn{1}{|c|}{$N_{h}$} & $\chi^{2}$ \\
\hline a) & $0.341 \pm 0.005$ & 0 & 96 \\
b) & 0 & $0.156 \pm 0.002$ & 878 \\
a)+b) & $0.319 \pm 0.012$ & $0.012 \pm 0.002$ & 91 \\
\hline
\end{tabular}

Table: The fitted normalizations $N_{s}$ and $N_{h}$ and the associated $\chi^{2}$ s (103 data points). The different cases considered are a) soft pomeron b) hard pomeron, and the linear combination a) + b).

We next consider scaling violations, both in the subasymptotic region of small $\sigma$ and small $\rho$, and in the post-asymptotic region of large $\rho$. This is best done by rescaling $F_{2}^{p}$ by a factor

$$
R_{F}(\sigma, \rho)=R \exp \left(-2 \gamma \sigma+\delta(\sigma / \rho)+\frac{1}{2} \ln \sigma+\ln (\rho / \gamma)\right)
$$

to remove all the leading behaviour in (2). The rescaled structure function should thus scale in both $\sigma$ and $\rho$ when both are sufficiently large to lie in the asymptotic region: $R_{F} F_{2}^{p}=N+O(1 / \sigma)+O(1 / \rho)$. This double asymptotic scaling behaviour is tested in the two scaling plots fig. 2, where we also display the predictions obtained [1] by applying the leading small- $x$ form of the evolution equations to a typical soft starting gluon distribution. Specifically, fig. 2a) shows that the scaling in $\sigma$ sets in very rapidly, as all the points on the plot lie in the asymptotic regime; fig. $2 \mathrm{~b}$ ) shows that the scaling in $\rho$ only sets in for $\rho^{2} \gtrsim 2$. However even if $\rho$ is as low as $\rho \sim \frac{1}{2}$ the subasymptotic corrections due to $f(\gamma / \rho)$ seem fairly well accounted for by the scaling violation displayed by the curves of fig. 2 .

More interestingly, at large $\rho$ there now appears to be a statistically significant rise above the scaling prediction. To test the significance of this rise, we fitted to the data a linear combination of the behaviour discussed above and displayed by the curves of fig. 2, and a "hard pomeron" behaviour, which violates scaling by rising with $\rho$ (see ref. [1] for a more detailed discussion). Including in the fit the 103 HERA points with both $\sigma^{2}$ and $\rho^{2}$ greater than one half gives the results displayed in the table. The data seem to prefer a $4 \pm 1 \%$ admixture of the hard pomeron solution. One should be very cautious about taking this as evidence for the hard pomeron per se, however, since higher loop corrections should give a similar rise [8]. It should be possible to settle this issue decisively when a more detailed set of data and more accurate theoretical calculations become available.

Acknowledgement: We are very grateful to Dick Roberts for communicating to us the data of refs [5:6]. 


\section{References}

[1] R.D. Ball and S. Forte, preprint CERN-TH.7265/94, hep-ph/9405320.

[2] ZEUS Collab.,Phys. Lett. B316 (1993) 412.

[3] H1 Collab., Nucl. Phys. B407 (1993) 515.

[4] NMC Collab., Phys. Lett. B295 (1992) 159.

[5] G. Wolf, talk at the International Workshop on Deep Inelastic Scattering. Eilat, Israel, February 1994; M. Roco, talk at the 29th Rencontre de Moriond, March 1994.

[6] K. Müller, talk at the 29th Rencontre de Moriond, March 1994.

[7] A. De Rujula, S.L. Glashow, H.D. Politzer, S.B. Treiman, F. Wilczek and A. Zee, Phys. Rev. D10 (1974) 1649; see also Yu.L. Dokshitzer, Sov. Phys. J.E.T.P. 46 (1977) 641.

[8] R.K. Ellis, Z. Kunszt, and E.M. Levin, Fermilab-PUB-93/350-T, ETH-TH/93-41, to be published in Nucl. Phys. B. 


\section{Figure Captions}

Fig. 1. Values of $R_{F}^{\prime} F_{2}^{p}$ plotted against $\sigma$ : diamonds are ZEUS data [5], squares H1 data [6], and crosses are NMC data. The best fit straight line is also shown.

Fig. 2. $R_{F} F_{2}^{p}$ plotted against a) $\sigma$ and b) $\rho$. Included in the plots are all the HERA data with $\rho>1.2, \sigma>0.7$, respectively. The curves show the prediction obtained [1] evolving a typical soft starting gluon distribution: a) dot-dash curve, $\rho=1.4$; solid curve, $\rho=2.2$; dotted curve, $\rho=3.2$. b) dot-dash curve, $\sigma=1.1$; solid curve, $\sigma=1.8$; dotted curve, $\sigma=2.1$. 
This figure "fig1-1.png" is available in "png" format from: http://arxiv.org/ps/hep-ph/9406385v3 
This figure "fig1-2.png" is available in "png" format from: http://arxiv.org/ps/hep-ph/9406385v3 
This figure "fig1-3.png" is available in "png" format from: http://arxiv.org/ps/hep-ph/9406385v3 


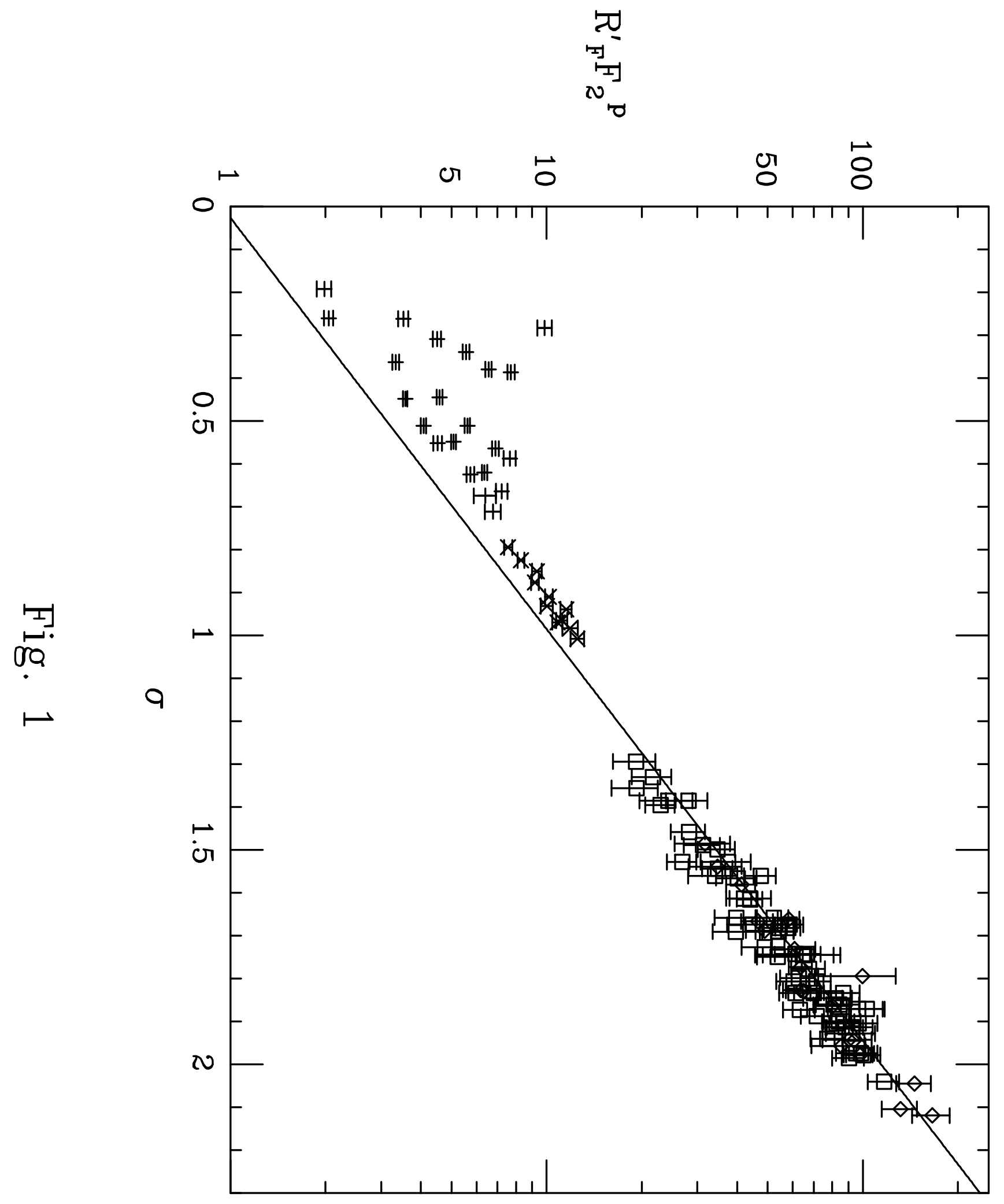




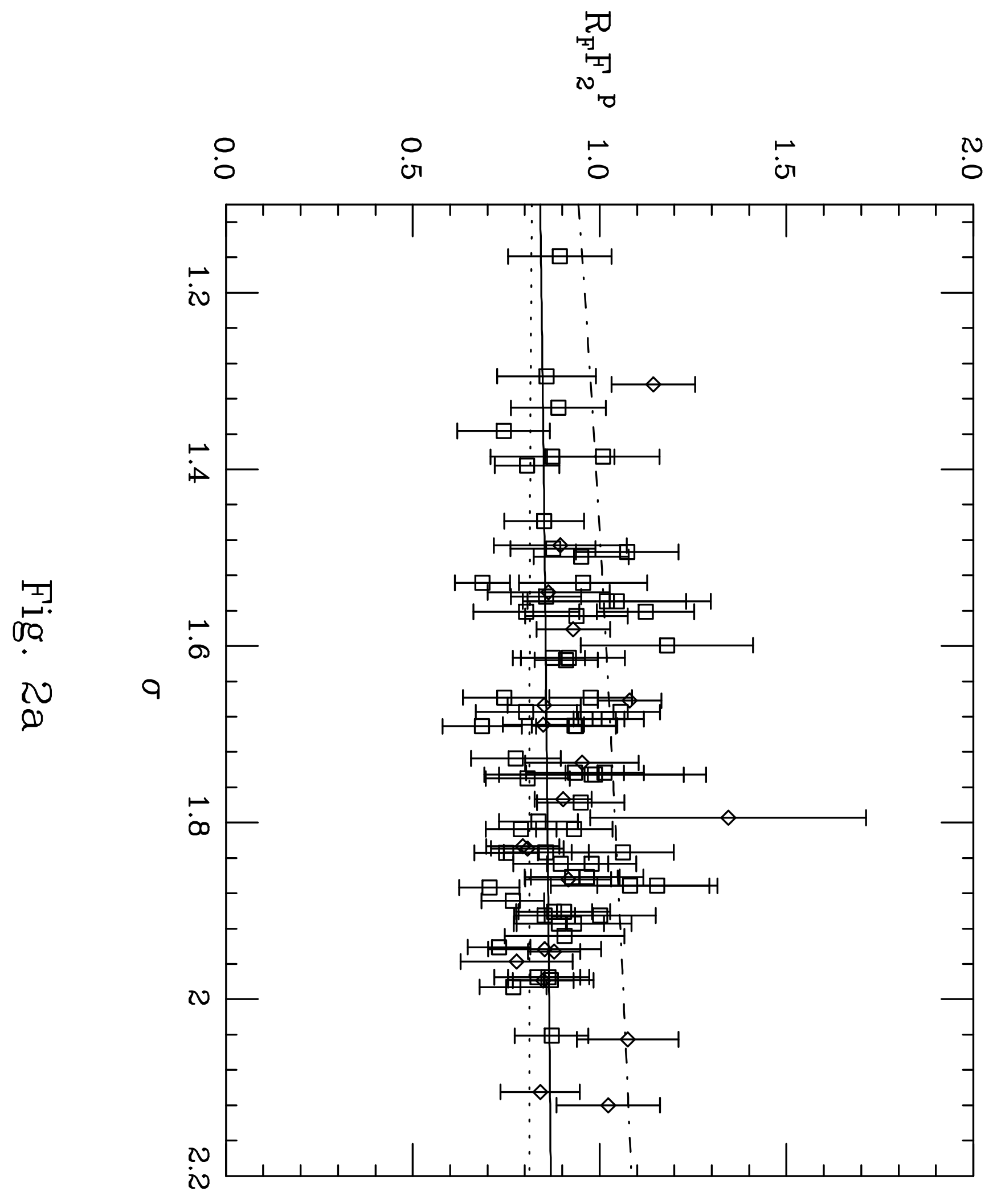




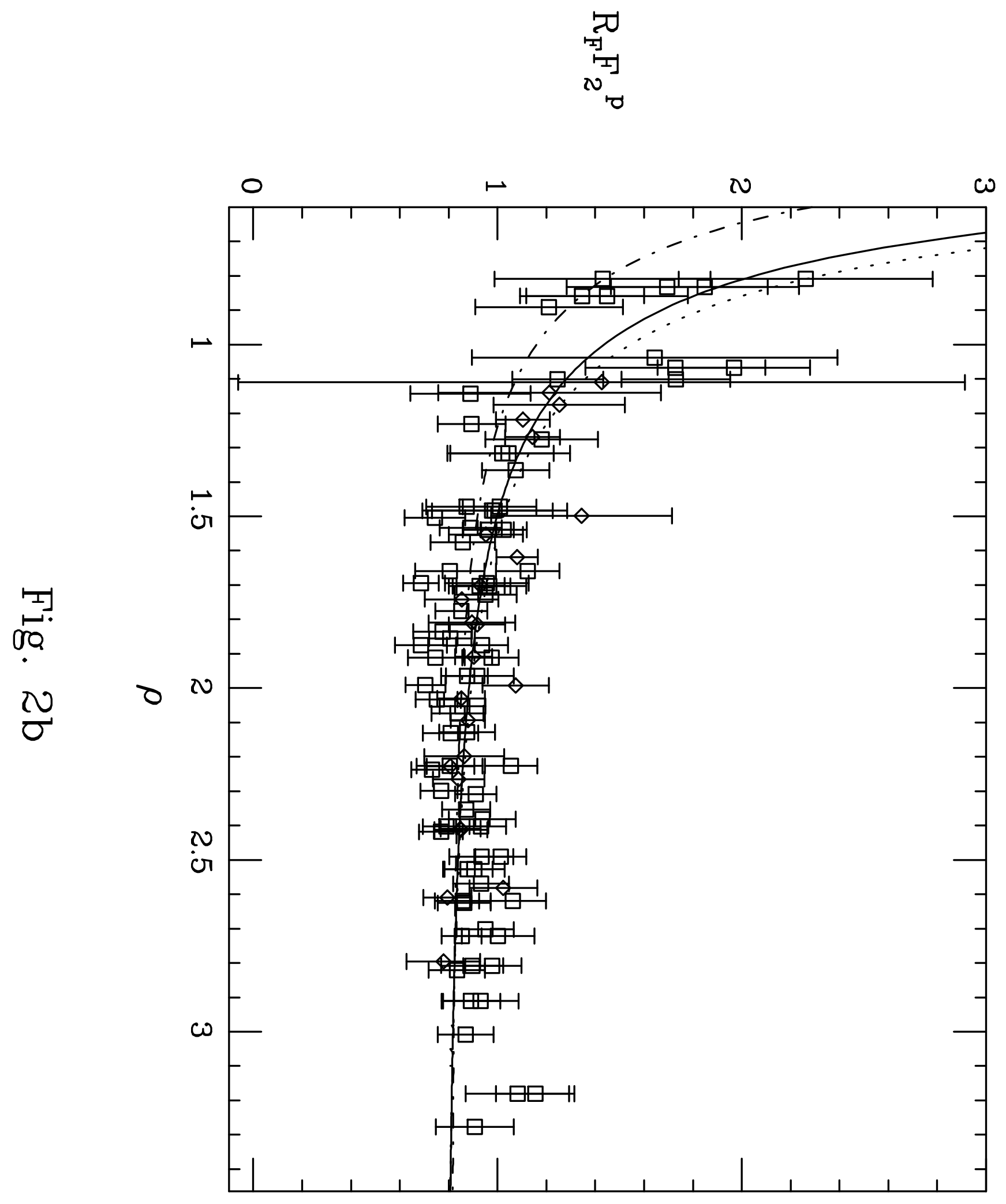

